\title{
A Real Time Visible Watermarking Technique using Dual Tree Complex Wavelet Transformation
}

\author{
Prodipta Bhowmik \\ Assistant Professor, Department of IT \\ Techno India, Salt Lake \\ Kolkata-700091, India
}

\author{
Tanmay Bhattacharya, PhD \\ Associate Professor, Department of IT \\ Techno India, Salt Lake \\ Kolkata-700091, India
}

\begin{abstract}
The key idea of this paper is to propose a real-time, selfauthenticating, watermarking technique. Here the watermark has been constructed from the image itself using Toeplitz matrix and the embedding region is selected by the user at real-time. Embedding region should be selected from Region of Non Interest (RONI) of the image. Dual Tree Complex Wavelet transformations (DT-CWT) based embedding technique has been applied for the embedding purpose. The key is shared or transferred to the receiver through a secret channel. At the receiving end, the extracted watermark is matched with the matrix generated from the bit-sequence (key) which ensures the originality of the image.
\end{abstract}

\section{Keywords}

Digital watermarking, Toeplitz matrix, DT-CWT, Image authentication.

\section{INTRODUCTION}

In today's world, the use of digital multimedia content has increased a lot. Images can be processed, used and shared very easily. It can be transmitted also. Hence there are risk of unauthorized access and modifications of digital content. So authentication procedure needs to be applied to secure digital contents. Watermarking is a technique to hide digital information into other digital contents. It verifies the authenticity or integrity of the carrier signal. A digital watermark should be robust with respect to various attacks if the embedded information may be detected reliably even if degraded by various noises. A watermarked signal is called imperceptible if it is equivalent to the original one. A self authentication technique is basically used to identify tamper detection. The digital watermarking can be categorized into two types mainly: Spatial domain and Frequency domain. Least significant Bit (LSB) [1] is the most common spatial domain technique. Intensities are modified for some selected pixels in this technique. It is not robust as it is prone to attacks by unauthorized users. In frequency domain technique [2], the image is transformed into the frequency domain and then watermark is embedded. Compared to LSB, it is more secured and robust to various attacks. In this approach, various transformation procedures are applied like Discrete Cosine Transformation (DCT) [3], Discrete Fourier Transformations (DFT) [4], Discrete Wavelet Transformation (DWT) [5], and Complex Wavelet Transformation (CWT) [6].

\section{PROPOSED METHOD}

There are three phases in this proposed scheme viz. Watermark generation, embedding, extraction and matching. Here the watermark is generated from the original image and the resolution of the watermark is on eighth of the original image.

\subsection{Watermark generation}

The Watermark is generated by the following procedure:

$>$ Let the original image $\mathrm{P}$ is of size $\mathrm{N} \times \mathrm{N}$

$>$ From $\mathrm{P}$, randomly N-1 elements have been selected so that all the pixels have valid $3 \times 3$ neighborhoods.

$>$ Let the neighbors are: $\mathrm{P}(\mathrm{x}-1, \mathrm{y}-1), \mathrm{P}(\mathrm{x}-1, \mathrm{y}), \mathrm{P}(\mathrm{x}-1, \mathrm{y}+1)$, $\mathrm{P}(\mathrm{x}, \mathrm{y}+1), \mathrm{P}(\mathrm{x}+1, \mathrm{y}+1), \mathrm{P}(\mathrm{x}+1, \mathrm{y}), \mathrm{P}(\mathrm{x}+1, \mathrm{y}-1), \mathrm{P}(\mathrm{x}, \mathrm{y}-1)$

$>$ Now mean value $\left[\right.$ say $\left.\mathrm{P}_{\mathrm{a}}(\mathrm{x}, \mathrm{y})\right]$ of those neighborhoods is calculated.

$>$ A binary sequence $\mathrm{B}_{\mathrm{i}}$ is obtained by applying the following condition:

$\mathrm{B}_{\mathrm{i}}=\left\{\begin{array}{cl}0 & \text { if } \mathrm{P}(\mathrm{x}, \mathrm{y})>\mathrm{P}_{\mathrm{a}}(\mathrm{x}, \mathrm{y}) \\ 1 & \text { otherwise }\end{array}\right.$

Where $\mathrm{i}=1,2,3 \ldots \ldots \mathrm{N}-1$

A $8 X 8$ Toeplitz matrix has been generated from the 8 bit binary sequence $B_{i}$ obtained above, which is used as the watermark to be embedded in the original image.

Toeplitz Matrix: Toeplitz matrices [7] are matrices having constant entries along their diagonals. Toeplitz matrices arise in many different theoretical and applicative fields, in the mathematical modeling of all the problems where some sort of shift invariance occurs in terms of space or of time. The Toeplitz structure may occur entry-wise, for one-dimensional problems, or block-wise, for two-dimensional problems, or even at several nested levels in multidimensional problems. Toeplitz matrices may be finite or even infinite according to the features of the problem that is modeled.

Given $2 \mathrm{n}-1$ numbers $\mathrm{a}_{\mathrm{k}}$, where $\mathrm{k}=-\mathrm{n}+1, \ldots-1,0,1, \ldots, \mathrm{n}-1$, a Toeplitz matrix is of the following form: 


$$
\left[\begin{array}{ccccc}
a_{0} & a_{-1} & a_{-2} & \cdots & a_{-\pi+1} \\
a_{1} & a_{0} & a_{-1} & \omega_{0} & \vdots \\
a_{2} & a_{1} & a_{0} & \sigma_{0} & a_{-2} \\
\vdots & \sigma_{0} & \sigma_{0} & \sigma_{0} & a_{-1} \\
a_{n-1} & \cdots & a_{2} & a_{1} & a_{0}
\end{array}\right] .
$$

the image using DT-CWT. Finally inverse DT-CWT is applied to obtain the watermarked image.

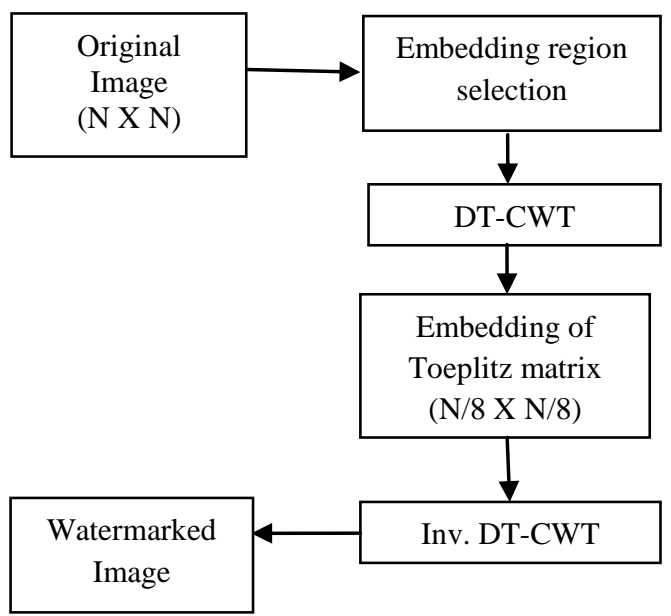

Fig 2. Watermark embedding process

Selected and corresponding embedded regions are shown in Fig. 3.
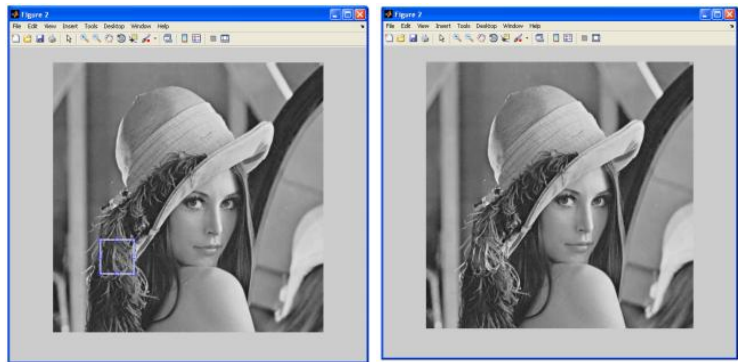

Fig 3. Original image with selected and embedded regions

\subsection{Watermark Extraction and Matching}

The received bit-sequence (key) is converted into $2 \mathrm{D}$ matrix (Toeplitz).The watermark is extracted from the watermarked image using the positions received from the sender through the secret channel.

Dual-tree wavelet transformations are applied to the watermarked image and then the embedded watermark is extracted.

The two watermarks (derived and extracted) are compared by applying the correlation. If the correlation is within the specified range, the watermarks are matched and authenticity is preserved, otherwise the received image is not the original one.

The entire watermark extraction and matching process is shown in Fig. 4. 


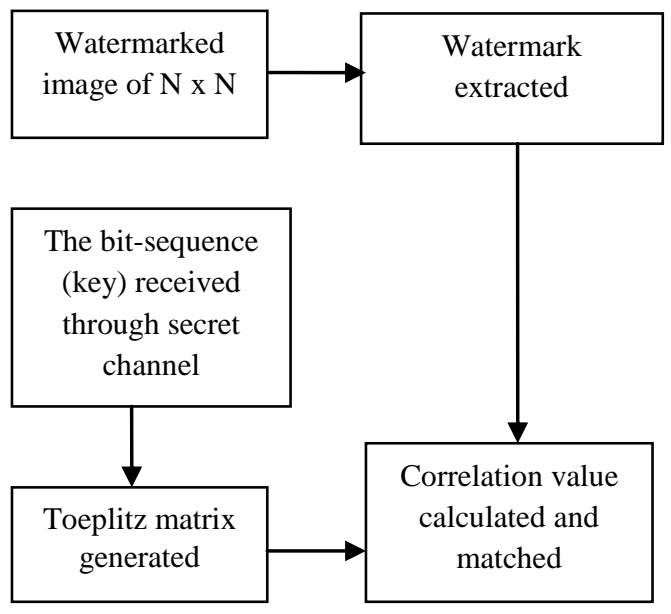

Fig 4. Watermark extraction

\section{EXPERIMENTAL RESULTS}

For testing, the size of original image (lena.jpg) is $512 \mathrm{X} 512$. A 64x64 Toeplitz matrix (binary watermark signal) has been constructed from original image and is embedded within the selected region. The proposed method has been implemented with MATLAB, 2012b and tested with various benchmark images. Table1 shows that PSNR and MAE values are within acceptable range i.e. visual difference between the original and watermarked images are within tolerable limit. Expressions for PSNR and MAE are given below where variables are of standard meaning:

$$
P S N R=10 \cdot \log _{10}\left(\frac{M A X_{I}^{2}}{M S E}\right)
$$

Where,

$$
\begin{aligned}
& M S E=\frac{1}{m n} \sum_{i=0}^{m-1} \sum_{j=0}^{n-1}[I(i, j)-K(i, j)]^{2} \\
& \text { MAE }=\frac{1}{n} \sum_{i=1}^{n}\left|f_{i}-y_{i}\right|=\frac{1}{n} \sum_{i=1}^{n}\left|e_{i}\right| .
\end{aligned}
$$

Table1: Imperceptibility Test

\begin{tabular}{|c|c|c|c|}
\hline Actual Image & $\begin{array}{c}\text { Watermarked } \\
\text { Image }\end{array}$ & PSNR & MAE \\
\hline & & & \\
\hline & & & \\
\hline
\end{tabular}

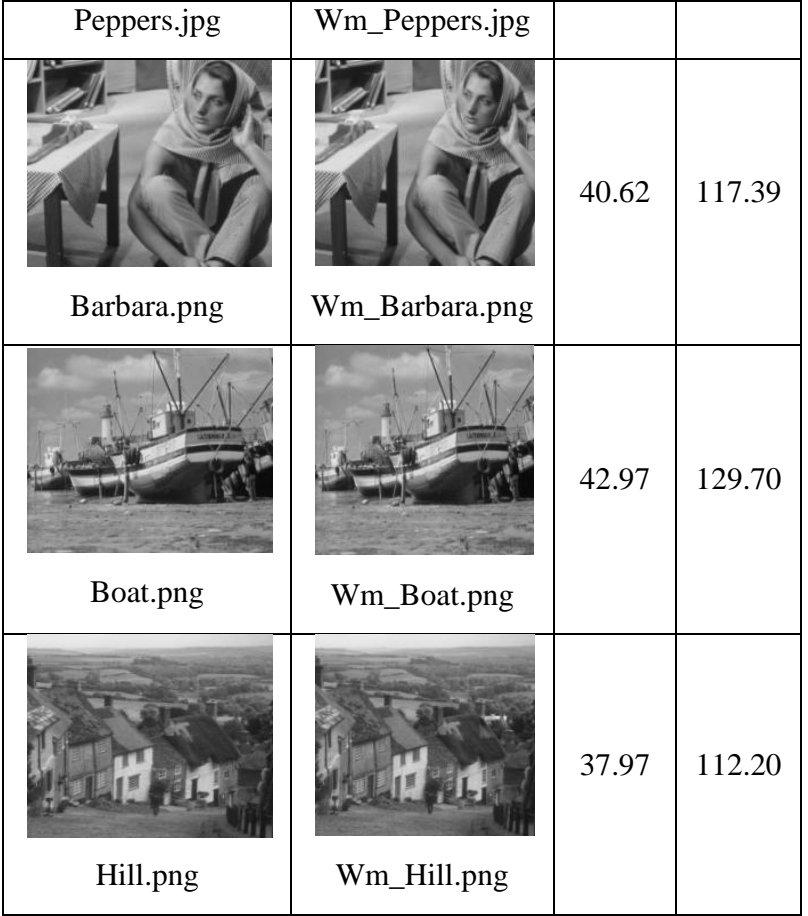

Table 2 exhibits high correlation value, except 'salt \& pepper' noise, between the derived and extracted matrices even after the addition of noises, which ensure the robustness of the

\begin{tabular}{|c|c|c|c|}
\hline Noise & $\begin{array}{c}\text { Generated } \\
\text { Watermark }\end{array}$ & $\begin{array}{c}\text { Extracted } \\
\text { Watermark }\end{array}$ & $\begin{array}{c}\text { Correlation } \\
\text { value }\end{array}$ \\
\hline Gaussian & & & 0.9976 \\
\hline Poisson & & & 0.9982 \\
\hline $\begin{array}{l}\text { Salt \& } \\
\text { Papper }\end{array}$ & & & 0.9434 \\
\hline & & & \\
\hline
\end{tabular}
proposed method.

Table2: Robustness against Noise (for 'boat.png') 


\section{CONCLUSION}

The proposed watermarking scheme provides a complete algorithm that embeds and extracts the watermark information effectively. The watermark pattern is session based as in every session it is constructed from the original image randomly. The scheme provides robustness as well as security by adopting real-time embedding approach. The performance of the watermarking scheme is tested with various noise attacks and where it performs well but the performance for salt \& pepper noise is not that so satisfactory.

\section{REFERENCES}

[1] C.I. Podilchuk, E.J. Delp "Digital watermarking: algorithms and applications, "Signal Processing Magazine, Vol 18, pp.33-46, IEEE, July 2001.

[2] D.R. Denslin Brabin, Dr. J.Jebamalar Tamilselvi, Dr. S. Janakiraman, "A Visible Watermarking Scheme for Digital Images in Frequency Domain" published in Int. J. Advanced Networking and Applications (IJANA) Volume: 04 Issue:03 Pages: 1635-1639 (2012) ISSN : 0975- 0290.

[3] Tao B. and Dickinson B., "Adaptive Watermarking in DCT Domain", Proc. of IEEE International Conf. on Acoustics, Speech and Signal Processing, ICASSP-97, Vol.4, pp.1985-2988, 1997.
[4] Awanish Kr Kaushik, A Novel Approach for Digital Watermarking of an Image Using DFT, published in International Journal of Electronics and Computer Science Engineering ISSN- 2277-1956/V1N1-35-41

[5] Nikita Kashyap, G. R. SINHA, Image Watermarking Using 3-Level Discrete Wavelet Transform (DWT), I.J. Modern Education and Computer Science, 2012, 3,50-56, Published Online April 2012 in MECS

[6] Natasa Terzua, Walter Geisselhardt, Digital Image Watermarking using Complex Wavelet Transform, Proceeding of the 2004 Workshop on Multimedia and Security, Pages 193-198,ISBN:1-58113-854-7

[7] Sathik, M.M., Sadakathullah Appa and Sujatha, S.S., "Application Of Toeplitz Matrix In Watermarking For Image Authentication", Published in Proceedings of International Conference on Computer, Communication and Electrical Technology (ICCCET), 2011, Tamilnadu, 18-19 March 2011, pp 55-59.

[8] Jayavani Adabala, K. Naga Prakash, DUAL TREE COMPLEX WAVELET TRANSFORM FOR DIGITAL WATERMARKING, International Journal of Advances in Engineering \& Technology, Sept 2012. CIJAET ISSN: 2231-1963

[9] DT-CWT based image fusion by VPS Naidu 05 July,2011, Mathworks. 